\title{
Perfil antropométrico e consumo alimentar de adolescentes de Teixeira de Freitas - Bahia ${ }^{1}$
}

\author{
Anthropometric profile and food intake of adolescents \\ in Teixeira de Freitas - Bahia, Brazil
}

\author{
Jailda Silva SANTOS2 \\ Maria Conceição Oliveira COSTA ${ }^{3}$ \\ Carlito Lopes NASCIMENTO SOBRINHO ${ }^{3}$ \\ Maria da Conceição Monteiro da SILVA ${ }^{2}$ \\ Karine Emanuelle Peixoto de SOUZA ${ }^{3}$ \\ Bianca Oliveira MELO3
}

R E S U M O

\section{Objetivo}

Descrever o perfil antropométrico e o consumo alimentar de adolescentes das escolas públicas de um município do Estado da Bahia.

\section{Métodos}

Estudo de corte transversal realizado com amostra aleatória por conglomerado de 354 alunos, dos 1678 adolescentes de dezessete a dezenove anos matriculados nas 23 escolas elegíveis do município. Foram coletados dados sobre freqüência de consumo alimentar qualitativo e indicadores antropométricos, como o índice de massa corporal; pregas cutâneas triciptal e subescapular e o indicador altura/idade, segundo a Organização Mundial da Saúde. Realizou-se análise bivariada e estratificada, sendo a significância estatística verificada pelo qui-quadrado e teste exato de Fischer, com nível crítico de $5 \%$.

\section{Resultados}

A análise antropométrica evidenciou maior prevalência de sobrepeso e obesidade (5,1\%) no sexo feminino e de magreza no masculino (6,4\%); o déficit de crescimento foi observado em $25,0 \%$ dos adolescentes, sendo $15,0 \%$ de leve a moderado e 10,0\% grave. Evidenciou-se também consumo habitual de arroz, feijão, farinha de mandioca, pão francês, manteiga, margarina, açúcar e café, sendo baixo o consumo de produtos lácteos,

\footnotetext{
1 Artigo elaborado a partir da dissertação de J.S. SANTOS, intitulada: "Perfil Antropométrico e Consumo Alimentar de Adolescentes das Escolas Públicas Municipais de Teixeira de Freitas - Bahia". Escola de Nutrição, Universidade Federal da Bahia. 2003.

2 Escola de Nutrição, Universidade Federal da Bahia. Salvador, BA, Brasil.

${ }^{3}$ Departamento de Saúde, Centro de Pós-Graduação em Saúde Coletiva, Universidade Estadual de Feira de Santana. Av. Universitária, km 03, Br 116, 44031-460, Campus Universitário, Módulo VI, Feira de Santana, BA, Brazil. Correspondência para/Correspondence to: M.C.O. COSTA. E-mail: <costamco@hotmail.com>.
} 
frutas, legumes e hortaliças; $96,6 \%$ dos adolescentes realizavam mais de três refeições diárias e mais de $80,0 \%$ alimentavam-se no domicílio.

\section{Conclusão}

Os resultados apontam a necessidade de implementação de estratégias de prevenção dos problemas nutricionais por meio de uma alimentação e um estilo de vida saudáveis, assim como de estudos sobre os possíveis fatores comprometedores do crescimento dos adolescentes.

Termos de indexação: adolescente, consumo alimentar, antropometria.

\section{A B S T R A C T}

\section{Objective}

The objective was to describe the anthropometric profile and food intake in adolescents from public schools in a municipality of Bahia, Brazil.

\section{Methods}

A cross-sectional experimental design was employed with a random conglomerate sample of 354 adolescents, 17 to 19 years old, from a total of 1678 students enrolled in 23 eligible schools of the municipality. Qualitative data on the frequency of food consumption and anthropometric indicators were collected. Body weight/ height index, triceps skeletal muscle and sub-scapular skinfold measurements, were carried out according to World Health Organization. Bivariate and stratified analyses were then carried out, and statistical significance at the $5 \%$ level verified using chi-square $(\chi 2)$ and the exact Fischer test.

\section{Results}

An analysis of the anthropometric indicators showed prevalence for overweight and obesity in $5.1 \%$ of the females and prevalence for underweight in $6.4 \%$ of the males. Growth deficiency was noted in $25.0 \%$ of the adolescents; the deficiency being classified as moderate in $15.0 \%$ and severe in $10.0 \%$ of the adolescents. The usual food intake consisted of cooked rice, beans, manioc flour, wheat bread, butter, coffee and sugar. A low consumption of dairy products, fruits, vegetables and greens was observed. $96.6 \%$ of the adolescents had three meals per day, which were consumed at home by $80.0 \%$ of those interviewed.

\section{Conclusion}

The results point to a need to implement preventive strategies for nutritional problems, recommending appropriate food intake and healthier life styles. Furthermore, possible factors inhibiting growth in the adolescents should be studied.

Indexing terms: adolescent, food intake, anthropometric.

\section{N T R O D U Ç Ã O}

De acordo com a Organização Mundial da Saúde (OMS)1 , os indicadores antropométricos devem ser utilizados na determinação do estado nutricional e de saúde de indivíduos e coletividades, sendo importantes no diagnóstico e acompanhamento da situação nutricional e crescimento corporal.

No diagnóstico do déficit de crescimento em adolescentes, tem sido recomendado o indicador altura/idade $\left(\mathrm{A} / \mathrm{I}^{2}\right.$, que pode ser utilizado para avaliar a desnutrição pregressa (stunted) ou a continuidade desse déficit (stunting) ${ }^{3}$.
Considerando a baixa sensibilidade do índice de massa corporal (IMC) ${ }^{3-5}$ na avaliação do excesso de tecido adiposo em adolescentes, a OMS 1 recomenda o IMC ou índice de Quetelet (peso $(\mathrm{kg})$ /altura $\left(\mathrm{m}^{2}\right)$ ) associado a medidas como espessura da prega cutânea tricipital (PCT) e prega cutânea subescapular (PCSE) para o diagnóstico do excesso de tecido adiposo ou obesidade. Pesquisa realizada no Brasil, em 1989, utilizando o IMC, detectou prevalência de sobrepeso entre os adolescentes de 7,6\%, apresentando-se maior $(10,5 \%)$ no sexo feminino ${ }^{6}$. 
Apesar da escassez de estudos mais recentes que evidenciem no âmbito populacional a tendência da situação nutricional dos adolescentes no país, estudos realizados em alguns municípios possibilitam verificar prevalências de sobrepeso significante nessa faixa etária, situação que apresenta alta prevalência em países desenvolvidos ${ }^{7}$. O controle e monitoramento do sobrepeso e obesidade na infância e adolescência contribuem para a diminuição do risco de alterações metabólicas, doenças cardiovasculares e vários outros problemas de saúde na fase adulta ${ }^{8}$. Quanto ao baixo peso, a depender da gravidade, tem-se detectado que, a médio e longo prazo, pode comprometer o crescimento e o desenvolvimento puberal ${ }^{9}$.

Em relação ao consumo alimentar, é consenso que, no século $X X$, mudanças ocorridas na estrutura familiar, como a inserção da mulher no mercado de trabalho, influenciaram o padrão alimentar, sendo incomuns refeições com horários definidos e cardápios elaborados para o consumo em família, especialmente nas grandes cidades, onde as atividades são exercidas em diferentes horários e contextos. Na atualidade, entre os determinantes do consumo alimentar, também se destaca, em diferentes culturas, a influência da globalização, mais perceptível nas zonas urbanas, onde se observa o consumo exagerado de alimentos industrializados a partir da influência norte-americana nos costumes de diferentes países $^{10}$.

Na adolescência, as práticas alimentares refletem valores apreendidos na família e entre amigos, além da influência da mídia ${ }^{11}$. Nessa fase, o indivíduo está mudando o corpo e buscando a imagem corporal idealizada, portanto, a alimentação inadequada pode levar a desequilíbrios nutricionais que podem interferir no crescimento e no estado de saúde.

Compreendendo a relevância desse tema, uma vez que, em última instância, alterações no perfil nutricional resultam do desequilíbrio entre $\mathrm{o}$ consumo alimentar e o gasto energético, o presente estudo tem como objetivo descrever o perfil antropométrico e de consumo alimentar e verificar possíveis associações entre estado antropométrico e sexo, entre os adolescentes participantes do estudo, para que estratégias de educação alimentar possam ser sugeridas a fim de difundir informações que orientem os adolescentes e a população em geral quanto ao consumo alimentar adequado às necessidades nutricionais.

\section{MÉTODOS}

Trata-se de um estudo epidemiológico de corte transversal, com amostragem aleatória por conglomerado, obtida a partir de uma população de 1678 alunos de 17 a 19 anos, matriculados em 23 escolas públicas municipais de Teixeira de Freitas, extremo sul da Bahia, no período de outubro a dezembro de 2001. Para o cálculo do tamanho da amostra foram utilizados dados fornecidos pela Secretaria de Educação, estimando-se em média 24 alunos por turma e 72 alunos por escola. O número de conglomerados amostrados, aleatoriamente, foi estimado por meio da expressão $n=m^{*} x$, $(n=$ tamanho $d a$ amostra, $x=$ número médio de alunos por escola e $\mathrm{m}=$ número de conglomerados a serem sorteados $)^{12}$. O cálculo realizado resultou em seis escolas, obtendo-se uma amostra de 400 adolescentes. Contabilizaram-se após a coleta dos dados $18 \%$ de perdas e um " $n$ " de 354 alunos. Foram excluídas grávidas, lactantes até o quarto mês, paraplégicos, portadores de doenças do esqueleto e aqueles que não aceitaram participar.

As variáveis foram subdivididas em sociodemográficas e econômicas (sexo, idade, classe econômica); antropométricas (A/; IMC/idade (P/A $\left.{ }^{2}\right) ; \mathrm{PCT} /$ idade; PCSE/idade); hábito alimentar: freqüência de consumo alimentar (habitual: $\geq 4$ vezes/semana e consumido por mais de $50 \%$ dos adolescentes; não habitual: $<4$ vezes/semana e consumido por menos de $50 \%$; raramente consumido: uma vez/mês, ocasiões festivas mensais ou anuais) ${ }^{13}$. A classificação econômica das famílias foi adaptada do Critério 
de Classificação Econômica Brasil (CCEB) (classes $A 1, A 2, B 1, B 2, C, D, E)$, cuja pontuação é indicada pela renda ${ }^{14}$.

O estado antropométrico foi avaliado a partir do IMC relacionado à idade (IMC/I), indicador de adequação do peso em relação à altura, utilizando-se como referência os dados do National Health and Nutrition Examination Survey (NHANES I), adaptado por Must et. al. ${ }^{15}$. Utilizou-se também como critério de classificação a recomendação da OMS ${ }^{1}$, que acrescenta as PCT e PCSE para classificar o adolescente com obesidade. No que refere ao indicador $A / l$ para avaliar o crescimento linear, foi adotado o padrão de referência do National Center Health Statistics (NCHS), revisado pelo Centers for Disease Control $(C D C)^{16}$, expresso em percentil, utilizando as classificações Leve/Moderada e Grave para aqueles adolescentes que apresentaram crescimento inadequado.

Foram realizadas duas aferições para cada medida antropométrica e a média foi utilizada na análise estatística, conforme a OMS ${ }^{1}$. Para aferir o peso, utilizou-se balança eletrônica calibrada, com capacidade para $150 \mathrm{~kg}$, admitindo-se variação mínima de $100 \mathrm{~g}$ entre duas medidas, com adolescentes descalços e usando roupas leves. A estatura foi aferida com estadiômetro portátil marca Seca, de dois metros, fixado à parede nivelada, com o adolescente descalço, a cabeça posicionada na posição de Frankfurt e sem adereços, admitindo variação máxima de $0,5 \mathrm{~cm}$ entre duas medidas. A PCT foi aferida no ponto médio, entre o acrômio e olécrano, na face póstero-lateral do braço não dominante, marcado com o braço fletido, formando um ângulo de $90^{\circ}$ com o cotovelo; a prega foi formada a um centímetro acima do ponto médio com o braço relaxado ao longo do corpo e o adolescente em posição ereta. A PCSE foi medida a um centímetro abaixo do ângulo inferior da escápula com a prega formando um ângulo de $45^{\circ}$ com a coluna vertebral. Para a realização das pregas foi utilizado o calibrador de Lange, admitindo-se uma variação máxima de um milímetro.
Os dados sobre consumo alimentar foram coletados com o questionário de freqüência alimentar qualitativo (QFCA), considerado de baixo custo e aplicável na análise do hábito alimentar do indivíduo ou grupos populacionais ${ }^{13}$. Para analisar o QFCA, utilizou-se classificação por grupos de alimentos (pães, cereais, raízes e tubérculos; hortaliças, verduras e legumes; frutas; leguminosas; carnes e ovos), segundo Philippi ${ }^{17}$, acrescido do grupo "diversos" que inclui algumas preparações e bebidas.

Foi investigada também a freqüência das refeições diárias (café da manhã, lanche da manhã, almoço, lanche da tarde, jantar e ceia) para verificar o ritmo alimentar dos adolescentes por intermédio da combinação de duas ou mais refeições diárias, considerando como adequada a realização de pelo menos três refeições diárias ${ }^{18}$. O local de realização das refeições foi também registrado. Para a coleta dos dados foi solicitado consentimento dos pais ou responsáveis, no caso dos menores de 18 anos, e o consentimento livre e informado para os maiores, segundo resolução 196/96 do Conselho Nacional de Saúde ${ }^{19}$ e conforme ofício 04/2002, protocolo 05/2002, do Comitê de Ética e Pesquisa da Universidade Estadual da Feira de Santana (UEFS).

Nas análises estatísticas utilizaram-se os testes do qui-quadrado $\left(\chi^{2}\right)$ e exato de Fischer, com nível crítico de $5 \%$. As análises bivariada e estratificada foram realizadas para medir a associação entre a variável sexo e os indicadores antropométricos (IMC, pregas cutâneas e AlI). Os dados foram analisados e processados utilizando-se os programas Epi Info v.6 e SPSS v.9, no NNEPA/UEFS.

\section{RES ULTA DOS}

Conforme resultados, verificou-se que a maioria dos adolescentes era do sexo masculino, com maior freqüência a idade de dezessete anos, estudantes do noturno que trabalham durante o dia (Tabela 1). 
Tabela 1. Indicadores sociodemográficos e econômicos* de adolescentes e famílias. Escolas Públicas Municipais de Teixeira de Freitas, BA, 2000.

\begin{tabular}{|c|c|c|}
\hline Indicadores & $\mathrm{n}$ & $\%$ \\
\hline \multicolumn{3}{|l|}{ Idade } \\
\hline 17 & 164 & 46,3 \\
\hline 18 & 125 & 35,3 \\
\hline 19 & 65 & 18,4 \\
\hline \multicolumn{3}{|l|}{ Sexo } \\
\hline Masculino & 218 & 61,6 \\
\hline Feminino & 136 & 38,4 \\
\hline \multicolumn{3}{|l|}{ Turno em que estudava } \\
\hline Matutino & 21 & 5,9 \\
\hline Noturno & 333 & 94,1 \\
\hline \multicolumn{3}{|l|}{ Chefe de família } \\
\hline Pai & 220 & 62,1 \\
\hline Mãe & 87 & 24,6 \\
\hline Outro responsável & 47 & 13,3 \\
\hline \multicolumn{3}{|l|}{ Grau de instrução do chefe de família } \\
\hline Analfabeto/Primário incompleto & 265 & 75,0 \\
\hline Primário completo/Ginasial incompleto & 59 & 16,7 \\
\hline Ginasial completo/Colegial incompleto & 25 & 7,1 \\
\hline Colegial completo/Superior incompleto & 3 & 0,8 \\
\hline Superior completo & 2 & 0,6 \\
\hline \multicolumn{3}{|l|}{ Posse de Bens } \\
\hline Rádio & 322 & 91,0 \\
\hline Televisão (TV) & 306 & 86,4 \\
\hline Geladeira & 292 & 82,5 \\
\hline Máquina de lavar & 152 & 42,9 \\
\hline Vídeo cassete & 61 & 17,2 \\
\hline Freezer & 49 & 13,8 \\
\hline Automóvel & 40 & 11,3 \\
\hline Moto & 2 & 0,6 \\
\hline \multicolumn{3}{|l|}{ Classe socioecocômica } \\
\hline B2 & 1 & 0,3 \\
\hline c & 15 & 4,3 \\
\hline D & 185 & 52,2 \\
\hline$E$ & 153 & 42,9 \\
\hline Total & 354 & 100,0 \\
\hline
\end{tabular}

Adaptado da Associação Nacional de Empresas de Pesquisa (ANEP) ${ }^{14}$.
Quanto à composição familiar e situação socioeconômica, observa-se, na Tabela 1, que $220(62,1 \%)$ famílias eram chefiadas pelo pai; $265(75,0 \%)$ chefes de família eram analfabetos ou tinham o curso primário incompleto; mais de 80,0\% das famílias possuíam rádio, TV e geladeira, menos de $20,0 \%$ possuíam vídeo cassete e freezer e apenas $11,3 \%$ possuíam automóvel. Segundo a classificação socioeconômica adotada, 185 (52,2\%) famílias encontravam-se na classe D, 153 (42,9\%) na classe $\mathrm{E}$ e apenas $16(4,6 \%)$ nas classes mais favorecidas (Tabela 1).

Em relação à classificação do estado antropométrico, de acordo com o IMC e pregas cutâneas, os resultados apontaram uma prevalência geral de magreza e de sobrepeso/ obesidade de $4,2 \%$ e $4,0 \%$, respectivamente, sendo maior a proporção de magreza no sexo masculino $(6,4 \%)$ e de sobrepeso/obesidade no sexo feminino $(5,1 \%)$, com diferenças estatisticamente significantes entre os sexos (Tabela 2).

Para o indicador $\mathrm{A} / \mathrm{l}$, os resultados indicaram que, em ambos os sexos, a freqüência de estatura adequada foi de $75,0 \%$, evidenciando que $25,0 \%$ apresentavam déficit do crescimento, sendo $15,3 \%$ leve a moderado e $9,6 \%$ grave, de acordo com o CDC ${ }^{16}$ (Tabela 3).

Dos alimentos consumidos habitualmente ( $\geq$ quatro vezes/semana, por mais de $50,0 \%$ dos adolescentes), destacaram-se o arroz e o feijão, consumidos por mais de $90,0 \%$ dos adolescentes.

Tabela 2. Classificação do estado antropométrico* de adolescentes, segundo o sexo. Escolas Públicas Municipais de Teixeira de Freitas, BA, 2000.

\begin{tabular}{|c|c|c|c|c|c|c|c|c|}
\hline \multirow{3}{*}{ Sexo } & \multicolumn{8}{|c|}{ Estado antropométrico } \\
\hline & \multicolumn{2}{|c|}{ Magreza } & \multicolumn{2}{|c|}{ Eutrófico } & \multicolumn{2}{|c|}{$\begin{array}{c}\text { Risco de sobrepeso e } \\
\text { obesidade }\end{array}$} & \multicolumn{2}{|c|}{ Total } \\
\hline & $n$ & $\%$ & $n$ & $\%$ & $n$ & $\%$ & $n$ & $\%$ \\
\hline Masculino & 14 & 6,4 & 197 & 90,4 & 7 & 3,2 & 218 & 100,0 \\
\hline Feminino & 1 & 0,7 & 128 & 94,1 & 7 & 5,1 & 136 & 100,0 \\
\hline Total & 15 & 4,2 & 325 & 91,8 & 14 & 4,0 & 354 & 100,0 \\
\hline
\end{tabular}

${ }^{*} \chi^{2}=7,31 ; p=0,0258$. 
Tabela 3. Classificação do estado antropométrico* de adolescentes segundo o sexo. Escolas Públicas Municipais de Teixeira de Freitas, BA, 2000.

\begin{tabular}{|c|c|c|c|c|c|c|c|c|}
\hline \multirow{3}{*}{ Sexo } & \multicolumn{8}{|c|}{ Estatura para idade } \\
\hline & \multicolumn{2}{|c|}{ Crescimento adequado } & \multicolumn{2}{|c|}{-RC leve a moderado } & \multicolumn{2}{|c|}{-RC grave } & \multicolumn{2}{|c|}{ Total } \\
\hline & $n$ & $\%$ & $n$ & $\%$ & $n$ & $\%$ & $n$ & $\%$ \\
\hline Masculino & 164 & 75,5 & 32 & 14,7 & 22 & 10,1 & 218 & 100,0 \\
\hline Feminino & 102 & 75,0 & 22 & 16,2 & 12 & 8,8 & 136 & 100,0 \\
\hline Total & 266 & 75,2 & 54 & 15,3 & 34 & 9,6 & 354 & 100,0 \\
\hline
\end{tabular}

$\chi^{2}=0,27 ; p=0,8755 ;{ }^{*} \mathrm{~A} / \mathrm{l}=\mathrm{Altura}(\mathrm{cm})$ em relação à idade (anos); $\bullet \mathrm{RC}=$ retardo de crescimento.

Tabela 4. Distribuição da freqüência de consumo alimentar segundo grupos de alimentos. Adolescentes das Escolas Públicas Municipais de Teixeira de Freitas, BA, 2000.

\begin{tabular}{|c|c|c|c|c|}
\hline \multirow{2}{*}{ Grupo de Alimentos* } & \multicolumn{2}{|c|}{$<4$ vezes/semana } & \multicolumn{2}{|c|}{$\geq 4$ vezes/semana } \\
\hline & $n$ & $\%$ & $n$ & $\%$ \\
\hline \multicolumn{5}{|c|}{ Pães, cereais, raízes e tubérculos } \\
\hline Arroz & 7 & 2,0 & 344 & 97,2 \\
\hline Pão francês & 72 & 20,3 & 231 & 65,3 \\
\hline Farinha de mandioca & 43 & 12,1 & 212 & 59,9 \\
\hline Pão doce & 89 & 25,1 & 172 & 48,6 \\
\hline Macarrão & 154 & 43,5 & 148 & 41,8 \\
\hline Biscoito doce & 148 & 41,8 & 97 & 27,4 \\
\hline Biscoito salgado & 141 & 39,8 & 92 & 26,0 \\
\hline Biscoito recheado & 167 & 47,2 & 70 & 19,8 \\
\hline Bolo & 154 & 43,5 & 68 & 19,2 \\
\hline Batata inglesa & 130 & 36,7 & 67 & 18,9 \\
\hline Pipoca & 121 & 34,2 & 60 & 16,9 \\
\hline \multicolumn{5}{|c|}{ Hortaliças, verduras e legumes } \\
\hline Tomate & 121 & 34,2 & 174 & 49,2 \\
\hline Alface & 129 & 36,4 & 113 & 31,9 \\
\hline \multicolumn{5}{|l|}{ Frutas } \\
\hline Suco de frutas & 157 & 44,4 & 141 & 39,8 \\
\hline Banana & 158 & 44,6 & 129 & 36,4 \\
\hline Laranja/tangerina & 165 & 46,6 & 117 & 33,1 \\
\hline Maçã & 152 & 42,9 & 71 & 20,1 \\
\hline Manga & 152 & 42,9 & 48 & 13,6 \\
\hline \multicolumn{5}{|l|}{ Leguminosas } \\
\hline Feijão & 16 & 4,5 & 333 & 94,1 \\
\hline \multicolumn{5}{|l|}{ Carnes e ovos } \\
\hline Carne de boi & 131 & 37,0 & 160 & 45,2 \\
\hline Ovos & 175 & 49,4 & 96 & 27,1 \\
\hline Frango & 215 & 60,7 & 79 & 22,3 \\
\hline \multicolumn{5}{|l|}{ Produtos lácteos } \\
\hline Leite integral & 96 & 27,1 & 130 & 36,7 \\
\hline \multicolumn{5}{|l|}{ Óleos e gorduras } \\
\hline Manteiga/margarina & 64 & 18,1 & 226 & 63,8 \\
\hline \multicolumn{5}{|l|}{ Doces } \\
\hline Açúcar & 34 & 9,6 & 287 & 81,1 \\
\hline Caramelos/balas & 113 & 31,9 & 171 & 48,3 \\
\hline \multicolumn{5}{|l|}{ Diversos } \\
\hline Café & 22 & 6,2 & 270 & 76,3 \\
\hline Refrigerante & 184 & 52,0 & 123 & 34,7 \\
\hline Suco em pó & 109 & 30,8 & 102 & 28,8 \\
\hline Salgados & 145 & 41,0 & 70 & 19,8 \\
\hline
\end{tabular}

*Adaptado de Philippi et al. ${ }^{18}$. 
Outros alimentos também foram considerados de consumo habitual: farinha de mandioca, pão francês, manteiga, margarina, açúcar e café (Tabela 4). Mais de 50,0\% dos adolescentes consumiam semanalmente alimentos como macarrão, pão doce e biscoitos, bolo, batata inglesa, pipoca, alface, tomate, banana, laranja ou tangerina, maçã, manga, suco de frutas, carne de boi, frango, ovos, leite integral, caramelos, salgados, sorvete e suco em pó (Tabela 4). Frango e refrigerante foram consumidos menos de quatro vezes na semana por $60,7 \%$ e $52,0 \%$ dos adolescentes, respectivamente, representando o tipo de carne e o item industrializado mais consumido.

Entre os alimentos raramente consumidos por mais de $70 \%$ dos adolescentes, nos diferentes grupos de alimentos, destacaram-se pão integral, milho verde, inhame, beterraba, pepino, abobrinha, couve-flor, vagem, abacate, pêra, uva, abacaxi, peixe enlatado e carne de porco, sendo que mais de 50\% raramente consumiam hambúrguer, peixe fresco, vísceras, lingüiça e salsicha. Os produtos lácteos eram raramente consumidos por $50 \%$ a $70 \%$, exceto o leite

Tabela 5. Distribuição do local das refeições e ritmo alimentar diário de adolescentes. Escolas Públicas Municipais de Teixeira de Freitas, BA, 2000.

\begin{tabular}{lcc}
\hline Local & $\mathrm{n}$ & $\%$ \\
\hline Casa & 300 & 85,2 \\
Casa/trabalho & 31 & 8,8 \\
Casa/lanchonete & 9 & 2,6 \\
Trabalho & 7 & 2,0 \\
Casa/restaurante & 3 & 0,9 \\
Restaurante & 1 & 0,3 \\
Lanchonete & 1 & 0,3 \\
\hline Total & $352 *$ & 100,0 \\
\hline Ritmos alimentares diários• & 12 & \\
Duas refeições & 77 & 21,8 \\
Três refeições & 130 & 36,7 \\
Quatro refeições & 102 & 28,8 \\
Cinco refeições & 33 & 9,3 \\
Seis refeições & 354 & 100,0 \\
\hline Total & & \\
\hline
\end{tabular}

*Dois questionários sem resposta; •Não houve nenhum caso para uma refeição diária. integral. Entre as gorduras, o bacon era consumido raramente por mais de $80 \%$ e, no grupo de diversos, o chocolate em barra e em pó eram consumidos por mais de $50 \%$ dos adolescentes.

Os resultados evidenciaram também que a maioria dos adolescentes, 342 (96,6\%), realizava três ou mais refeições por dia, desses, 130 (36,7\%) faziam quatro refeições/dia; 102 (28,8\%) faziam cinco refeições/dia; 77 (21,8\%) faziam seis refeições/dia e apenas 33 (9,9\%) realizavam três refeições diárias. Em relação ao local, $300(85,2 \%)$ realizavam as refeições no domicílio (Tabela 5).

\section{DISCUSSÃ O}

No Brasil, segundo dados da Associação Nacional de Empresas de Pesquisa (ANEP) ${ }^{14}$, a maior parte das famílias pertence às classes socioeconômicas de menor renda (D: 33\% e E: $31 \%)$, sendo que, nas Regiões Norte e Nordeste, a situação é mais precária, com $67 \%$ das famílias pertencentes às classes D e E (37\% e 30\%, respectivamente). Esses dados mostram que mais de $60 \%$ das famílias brasileiras se encontram em classes socioeconômicas menos favorecidas ${ }^{20}$.

Neste estudo, realizado em um município do nordeste brasileiro, com amostra de alunos das escolas públicas, os resultados corroboram estudos anteriores realizados no Brasil ${ }^{21}$, mostrando que mais de $90 \%$ das famílias dos adolescentes avaliados pertenciam às classes econômicas menos favorecidas ( $D$ e E) (Tabela 1). A inserção socioeconômica nas classes $D$ e $E$ na quase totalidade das famílias dos adolescentes pode ser um fator de explicação para o grande percentual - mais de $60 \%$ - de adolescentes inseridos no mercado de trabalho, a fim de contribuir com o aumento da renda familiar.

Em relação ao estado antropométrico, vários estudos municipais e nacionais têm evidenciado maior prevalência de excesso de peso entre adolescentes do sexo feminino ${ }^{22,23}$. Destaca-se, no âmbito nacional, a Pesquisa Nacional de 
Saúde e Nutrição (PNSN), realizada em 1989, que mostrou maior prevalência de sobrepeso, aproximadamente duas vezes maior $(10,5 \%)$ entre adolescentes do sexo feminino em relação ao masculino $(4,8 \%)^{6,22}$. Apesar da pouca diferença entre os sexos, estudo realizado em escolas da Vila Mariana, São Paulo, SP, detectou também maior prevalência de sobrepeso e obesidade $(17,0 \%)$ no sexo feminino em relação ao masculino $(15,0 \%)^{23}$. Os resultados do presente estudo concordam com os achados do PNSN ${ }^{22} \mathrm{e}$ ratificam outros estudos ${ }^{6,23}$. Entretanto, outras pesquisas mostraram resultados discordantes, com maior prevalência de sobrepeso e obesidade no sexo masculino em relação ao feminino ${ }^{7,24}$.

Existe consenso quanto ao padrão diferenciado do estado antropométrico entre os sexos. Sabe-se que, na puberdade, ocorre maior crescimento de tecido muscular no sexo masculino pela ação da testosterona e de tecido adiposo, no sexo feminino, pela ação do estrogênio ${ }^{25,26}$, entretanto, os fatores comportamentais, principalmente aqueles relacionados à alimentação e ao estilo de vida, têm contribuído para a ocorrência de sobrepeso e obesidade 9,22. $^{9}$. Considerando a diversidade de fatores que podem interferir nesse processo, é importante ressaltar que, na adolescência, os sexos diferem na idade de início e final do desenvolvimento puberal e na intensidade do estirão e desaceleração do crescimento, assim como quanto às diferenças ambientais (hábito alimentar, padrão de utilização de alimentos pela família e prática de atividade física) ${ }^{7,9,11}$. Dessa forma, é possível destacar alguns fatores que podem estar associados à baixa prevalência de obesidade entre os adolescentes deste estudo: mais de $80 \%$ realizavam refeições no próprio domicílio; a maioria deles trabalhava, condicionando maior gasto energético; e baixo consumo de produtos industrializados.

Em termos antropométricos, os critérios de diagnósticos e as tabelas de referências utilizadas para o adolescente, ainda em estudo, não expressam de forma precisa as modificações que ocorrem nesse período, principalmente durante a pubescência. Nessa perspectiva, Monteiro et al. ${ }^{4}$ e Chiara et al. ${ }^{5}$, ao estudarem o desempenho dos critérios diagnósticos utilizados para identificar adolescentes de quinze a dezesseis anos com sobrepeso, evidenciaram que o IMC, critério recomendado pela OMS, classificou erroneamente $9 \%$ a $15 \%$ dos homens e $12 \%$ a $17 \%$ das mulheres. Entretanto, apesar da baixa sensibilidade desse critério, a especificidade a ele conferida faz com que seja considerado um indicador adequado para a triagem de adolescentes com problemas nutricionais, como a obesidade.

No que se refere à estatura final, sabe-se que é resultado da interação e influência de fatores genéticos e ambientais. Segundo Monteiro et al. ${ }^{4}$, variações estaturais podem refletir problemas nutricionais associados às diferenças socioeconômicas entre grupos populacionais, sendo comum a presença de déficit estatural em adolescentes brasileiros de classes econômicas menos favorecidas. Eisenstein ${ }^{9}$ considera que, no Brasil, o diagnóstico de desnutrição é diferencial obrigatório para a avaliação de adolescentes com problemas de crescimento e atraso puberal. Dessa forma, analisar o crescimento linear implica considerar, além dos fatores hereditários, a história nutricional (desnutrição pregressa) e alimentar, doenças, atividade física, estresse e urbanização, principalmente quando essa influência é exercida nos períodos de maior velocidade de crescimento 27,28 .

Na presente pesquisa, foi identificado que mais de $90 \%$ dos adolescentes pertenciam às classes de baixo nível socioeconômico ( $D$ e E) (Tabela 1), portanto, é possível que esse fator tenha contribuído para a alta prevalência de adolescentes com déficit de crescimento (Tabela 3).

Sabe-se que o consumo e os hábitos alimentares da família são influenciados, entre outros fatores, pelas questões culturais, avanços tecnológicos na produção de alimentos, processo de industrialização, propagandas veiculadas pelos meios de comunicação e, principalmente, pela condição socioeconômica. Pesquisa sobre hábitos e práticas alimentares realizada entre donas de casa de São Paulo, em 1993, verificou maior diversificação alimentar e consumo de frutas, 
legumes e alimentos industrializados com o aumento da renda. Segundo esse estudo, o baixo consumo de frutas e legumes em famílias de baixa renda é devido à impossibilidade de compra, enquanto que em famílias com maior recurso está associado à falta de hábito ${ }^{29}$.

Outros estudos relacionados ao consumo alimentar de adolescentes têm também indicado baixa ingestão de produtos lácteos, frutas, hortaliças, alimentos fontes de proteína e ferro, concomitantemente ao elevado consumo de açúcar e gordura ${ }^{29,30}$. Em Teixeira de Freitas, os resultados foram semelhantes, tendo sido verificado também que frutas, legumes e hortaliças não faziam parte do consumo habitual dos adolescentes avaliados, demonstrando que o padrão alimentar qualitativo necessita de ajustes para prevenir deficiências nutricionais específicas que podem repercutir na saúde e estatura final. Uma possível explicação para essa questão decorre da contestação do padrão alimentar da família, comportamento esperado na adolescência ${ }^{28-30}$. No caso do baixo consumo dos produtos lácteos, também verificado neste estudo, é possível que estejam sendo substituídos por alimentos como café, consumido habitualmente, e refrigerante, consumido semanalmente, por mais de $50 \%$ dos adolescentes.

Na população brasileira, o feijão é considerado alimento de consumo habitual. Ressalta-se a importância do seu consumo juntamente com o de arroz, fornecendo aminoácidos como lisina, treonina, metionina e triptofano, importante aporte protéico vegetal, principalmente quando a proteína animal não faz parte do consumo habitual ${ }^{23}$. No presente estudo, o feijão foi o segundo alimento mais consumido, sendo superado apenas pelo arroz, rico em carboidrato; entretanto, existe a possibilidade de que a biodisponibilidade do feijão com relação ao ferro esteja comprometida, uma vez que foi detectada, também neste estudo, a baixa freqüência do consumo de frutas, fonte importante de vitamina $C$.

A maioria dos adolescentes desta pesquisa, realiza três ou mais refeições diárias, o que pode ser reflexo da predominância de refeições realizadas no domicílio (80\%).

\section{CONCLUSÃO}

Mais de $90 \%$ dos adolescentes pertenciam às classes socioeconômicas menos favorecidas $(\mathrm{D}$ e E); estudavam à noite e trabalhavam de dia.

O estado antropométrico diferiu entre os sexos, com percentual de magreza mais elevado entre os homens e de sobrepeso e obesidade entre as mulheres; $15 \%$ dos adolescentes apresentaram déficit de crescimento leve a moderado e 10\% grave.

O hábito alimentar dos adolescentes foi adequado em qualidade, número e local das refeições, muito embora não tenha sido habitual o consumo de frutas, legumes e produtos lácteos.

As determinações do perfil antropométrico e do consumo alimentar da população adolescente constituem importante subsídio às políticas de saúde e intervenções educativo-terapêuticas nas escolas, serviços e comunidade, com vistas a prevenir a ocorrência precoce de doenças metabólicas decorrentes da inadequação alimentar, justificando a necessidade de implementação de políticas sociais e econômicas que garantam a inclusão sistemática de práticas e hábitos alimentares saudáveis desde as primeiras séries do ensino fundamental.

\section{REFERÊ NCIAS}

1. World Health Organization. Physical status: the use and interpretation of athropometry. Geneva: WHO; 1995. Technical Report Series.

2. Engstrom EM. SISVAN: Instrumento para o combate aos distúrbios nutricionais em serviços de saúde: o diagnóstico nutricional. 2.ed. Rio de Janeiro: Fiocruz; 2002.

3. Sigulem DM, Devincenzi MU, Lessa AC. Diagnóstico do estado nutricional da criança e do adolescente. J Pediatr. 2000; 76 Suppl 3:S275-84.

4. Monteiro PO, Victora CG, Barros FC, Tomasi E. Diagnóstico de sobrepeso em adolescentes: estudo do desempenho de diferentes critérios para o IMC. Rev Saúde Pública. 2000; 34(5):206-13. 
5. Chiara V, Sichieri R, Martins PD. Sensibilidade e especificidade de classificação de sobrepeso em adolescentes. Rev Saúde Pública. 2003; 37(2):226-31. Erratum in Rev Saúde Pública. 2003; $37(6): 868$.

6. Coitinho $D C$, Leão $M M$, Recine $E$, Sichieri R. Condições nutricionais da população brasileira: adultos e idosos. In: Pesquisa Nacional de Saúde e Nutrição. Brasília: Ministério da Saúde; 1991.

7. Fonseca VM, Sichieri R, Veiga GV. Fatores associados à obesidade em adolescentes. Rev Saúde Pública. 1998; 32(6):541-9.

8. World Health Organization. Obesity: Preventing and managing the global epidemic. Geneva: WHO; 1998. Report of a WHO Consultation on obesity. 3-5 june; 1997.

9. Eisenstein E. Atraso puberal e desnutrição crônica primária [tese]. São Paulo: Escola Paulista de Medicina, Universidade Federal de São Paulo; 1999.

10. Bleil SI. O padrão alimentar ocidental: considerações sobre a mudança de hábitos no Brasil. Cad Debate. 1998; 6(1):1-24.

11. Branen L, Flecher J. Comparison of college student's current eating habits and recollections of their childhood food practices. J Nutr Educ. 1999; 31(6):304-9.

12. Cochian W. Sampling Technique. New York: John Wiley \& Sons; 1975. p.516-34.

13. Willett WC. Future directions in the development of food-frequency questionnaires. Am J Clin Nutr. 1994; 59(1 Suppl)):171S-4S.

14. Associação Nacional de Empresas de Pesquisa. Classificação Econômica Brasil do ANEP - [periódico eletrônico] 2002 [citado em 2002 maio 5]. Disponível em: http://www.anep.org.br/m.arquivol

15. Must A, Dallal GE, Dietz WH. Reference data for obesity: 85th 95th percentiles of body mass index (wt/ht2) and triceps skinfold thickness. Am J Clin Nutr. 1991; 53(4):839-46. Erratum in: Am J Clin Nutr. 1991; 54(5):773.

16. Center Diseases Control. National Center Health Statistics-CDC/NCHS, Growth Charts; 2000. p.28. Advance, no 314, december; 2000.

17. Philippi ST, Laterza AR, Cruz ATR, Ribeiro LC. Pirâmide alimentar adaptada: guia para escolha dos alimentos. Rev Nutr. 1999; 12(1):65-80

18. Philippi ST, Colucci ACA, Cruz ATR, Ferreira MN, Coutinho RL. Alimentação saudável na infância e adolescente. In: Curso de atualização em alimentação e nutrição para professores da rede pública de ensino. Escola Superior de Agricultura Luiz de Queiroz; 2000; Piracicaba, São Paulo. Piracicaba: ESALQ; 2000. p.46-60.
19. Brasil. Ministério da Saúde, Conselho Nacional de Saúde. Diretrizes e normas regulamentadoras de pesquisas envolvendo seres humanos: resolução 196/96 do Conselho nacional de Saúde. Rio de Janeiro: Ministério da Saúde Fundação Osvaldo Cruz; 1998.

20. Instituto Brasileiro de Geografia e Estatística. Estudo Nacional de Despesa Familiar - ENDEF. Tabela de composição de alimentos. 4.ed. Rio de Janeiro: IBGE; 1996.

21. Gambardella AM, Goltieb SL. Dispêndio energético de adolescentes do período noturno. Rev Saúde Pública. 1998; 32(5):1-9.

22. Neutzling MB. Sobrepeso em adolescentes brasileiros - Brasil, PNSN-1989 [tese]. São Paulo: Escola Paulista de Medicina, Universidade Federal de São Paulo; 1998.

23. Gama CM. Consumo alimentar e estado nutricional de adolescentes matriculados em escolas da rede particular e estadual do bairro de Vila Mariana, São Paulo [tese]. São Paulo: Escola Paulista de Medicina, Universidade Federal de São Paulo; 1999.

24. Vieira VCR, Priore SE, Ribeiro SMR, Franceschini SCC, Almeida LP. Perfil socioeconômico, nutricional e de saúde de adolescentes recém ingressos em uma universidade pública. Rev Nutr. 2002; 15(3):273-8.

25. Marshall WA, Tanner JM. Puberty. In: Falkner F, Tanner JM. The human growth: A comprehensive treatise. 2nd ed. New York: Plenum Press; 1986. p.171-210.

26. Costa MCO. Crescimento e desenvolvimento na infância e adolescência. In: Costa MCO, Souza RP. Avaliação e cuidados primários da criança e do adolescente. Porto Alegre: Artes Médicas; 1998. p.35-55.

27. Waterlow JC. Classification and definition of protein-caloric malnutrition. In: Beaton $\mathrm{GH}$, Bengoa JM. Nutrition in preventive medicine. Geneve: WHO; 1976. p.530-50.

28. Priore SE. Composição corporal e hábitos alimentares de adolescentes: uma contribuição à interpretação de indicadores nutricionais [tese]. São Paulo: Universidade Federal de São Paulo; 1998.

29. Oliveira SPM, Annie T. Hábitos e práticas alimentares em três localidades da cidade de São Paulo (Brasil). Nutr Rev. 1998; 11(1):37-50.

30. Gambardella AMD, Frutuoso MFP, Franchi C. Prática alimentar de adolescentes. Rev Nutr. 1999; 12(1):55-63

Recebido para publicação em 25 de março e aceito em 7 de julho de 2004 